\title{
Three port logic gate using forward volume spin wave interference in a thin yttrium iron garnet film
}

Taichi Goto $\mathbb{1}^{1,2,3^{*}}$, Takuya Yoshimoto ${ }^{1}$, Bungo Iwamoto $^{1}$, Kei Shimada ${ }^{1}$, Caroline A. Ross $\mathbb{D}^{3}$, Koji Sekiguchi ${ }^{4}$, Alexander B. Granovsky ${ }^{5}$, Yuichi Nakamura ${ }^{1}$, Hironaga Uchida ${ }^{1}$ \& Mitsuteru Inoue ${ }^{1,6}$

We demonstrate a logic gate based on interference of forward volume spin waves (FVSWs) propagating in a $54 \mathrm{~nm}$ thick, $100 \mu \mathrm{m}$ wide yttrium iron garnet waveguide grown epitaxially on a garnet substrate. Two FVSWs injected by coplanar waveguides were made to interfere constructively and destructively by varying their phase difference, showing an XNOR logic function. The reflected and resonant waves generated at the edges of the waveguide were suppressed using spin wave absorbers. The observed isolation ratio was $19 \mathrm{~dB}$ for a magnetic field of $2.80 \mathrm{kOe}\left(=223 \mathrm{kA} \mathrm{m}^{-1}\right)$ applied perpendicular to the film. The wavelength and device length were $\sim 8.9 \mu \mathrm{m}$ and $\sim 53 \mu \mathrm{m}$, respectively. Further, the interference state of the SWs was analyzed using three-dimensional radio frequency simulations.

Wave-based devices enable non-Boolean operations and are attractive for next-generation information processing. Magnonic circuits based on spin waves $(\mathrm{SWs})^{1-8}$ show great potential in this regard because of their low Joule heating as well as the wide range of tunability $(>1 \mathrm{~mm})$ of the wavelength of SWs, which can be varied by changing the waveguide and/or the antenna design. The footprint of devices based on SW interference can be much smaller than that of existing the radio frequency (RF) devices because of the sub-millimeter wavelength of SWs.

SWs have already been used successfully for forming logic gates to produce two- ${ }^{9,10}$, three- ${ }^{11-13}$, and four-port ${ }^{14,15}$ devices. These devices showed NOT, XOR, XNOR, NAND and NOR logic gate functionalities. The functions of the four-port NAND and NOR gates were switchable by adjusting the phase of the additional SW input ${ }^{14}$, and additionally, these devices could be used as a three-input majority gate ${ }^{15}$. These devices are based on the interference of electromagnetic waves (EMW) $)^{9,12}$ or that of SW ${ }^{10,11,13-15}$. Furthermore, magnonic crystals $^{12,16-39}$ can be incorporated into SW devices as filters ${ }^{29,32}$ and modulators ${ }^{26,30,40}$. Therefore, all logic gate functions for realizing an arithmetic logic unit have been demonstrated individually so far. The next step in the development of SW integrated circuits (ICs) is combining individual devices to demonstrate concatenation and more complex functionality.

There are three key requirements for the development of SW ICs. First, the SWs should have a large propagation length (at least an order of magnitude longer than the SW wavelength), i.e., the Gilbert damping factor should be low. This propagation length limits the number of logic gates that can be concatenated without amplification. The material with the lowest damping is yttrium iron garnet ( $\mathrm{YIG}, \mathrm{Y}_{3} \mathrm{Fe}_{5} \mathrm{O}_{12}$ ). In comparison, other materials e.g., Heusler alloys ${ }^{41,42}$, permalloy ${ }^{10,13,43-45}$, and $\mathrm{CoFeB}^{46}$ have damping at least an order of magnitude higher. However, the preparation of $\mathrm{YIG}^{47-67}$ and etching or liftoff processes that preserve the properties of the YIG $^{60,61,66,68-70}$ present challenges, particularly for integration on a semiconductor platform. Second, to efficiently interconnect SW devices, the waveguides need to be curved or bent. This necessitates the use of forward volume (FV) SWs because this is the only SW showing in-plane uniformity ${ }^{14,71}$. The other two SW modes, i.e., backward volume SWs and surface SWs, change their wavelengths when the propagation direction is changed because the angle between the wavevector and magnetic field varies. However, FV SWs suffer from noise generated at the

\footnotetext{
${ }^{1}$ Department of Electrical and Electronic Information Engineering, Toyohashi University of Technology, 1-1 Hibarigaoka, Tempaku, Toyohashi, Aichi, 441-8580, Japan. ${ }^{2}$ JST, PRESTO, 4-1-8 Honcho, Kawaguchi, Saitama, 332-0012, Japan. ${ }^{3}$ Department of Materials Science and Engineering, Massachusetts Institute of Technology, 77 Massachusetts Avenue, Cambridge, Massachusetts, 02139, USA. ${ }^{4}$ Faculty of Engineering, Yokohama National University, 79-5 Tokiwadai, Hodogaya, Yokohama, Kanagawa, 240-8501, Japan. ${ }^{5}$ Faculty of Physics, Moscow State University, Leninskie Gory, Moscow, 119992, Russia. ${ }^{6}$ Department of Physical Electronics and Technology, St. Petersburg Electrotechnical University, St. Petersburg, 197376, Russia. *email: goto@ee.tut.ac.jp
} 
(a)

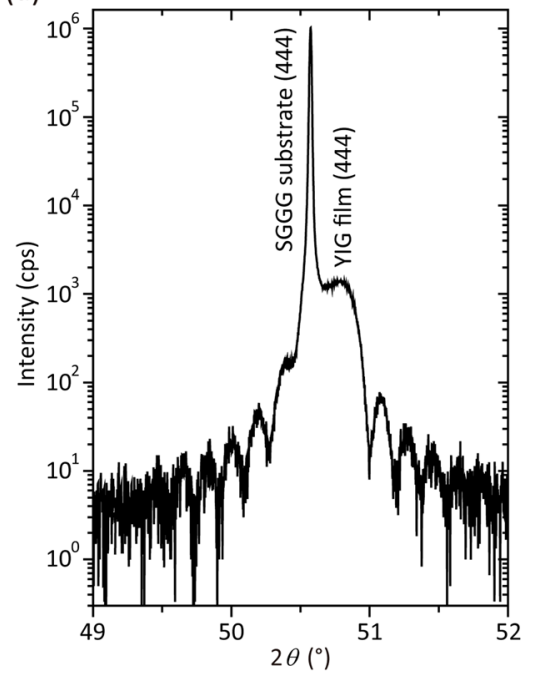

(b)

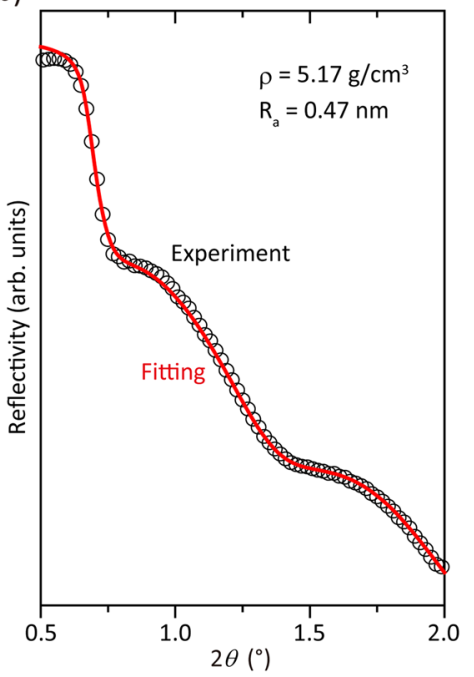

(c)

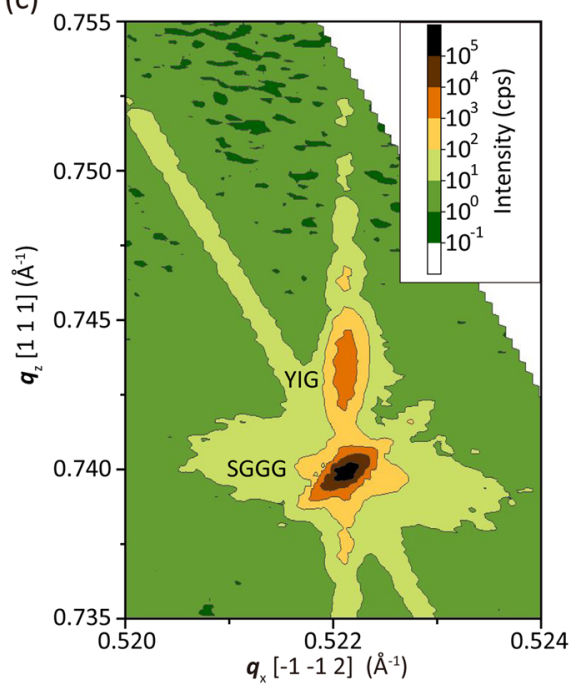

Figure 1. (a) $2 \theta-\omega$ XRD of YIG film grown on SGGG substrate. (b) XRR of (111)-oriented YIG. (c) RSM in the vicinity of the (880) peak.

waveguide edge or boundary reflections and strong standing modes ${ }^{11,71-73}$. Finally, when the thickness of the YIG film and the sizes of the antennas are on the order of a millimeter or sub-millimeter, as used in previous logic gates $^{7,9,11,14,15,74}$, the wavelengths of SWs are on a millimeter or sub-millimeter scale resulting in device footprints on the order of $\mathrm{mm}^{2}$. Antennas with micron to sub-micron lengthscales can excite short SWs with wavelengths on the similar lengthscales ${ }^{13,45,59,61,65}$. This motivates the development and integration of devices based on thinner YIG films and an antenna with smaller dimensions.

In this study, we have addressed these challenges to successfully demonstrate the smallest three-port XNOR gate to date. The device footprint area was four orders of magnitude smaller than previous work ${ }^{11}$ and the performance was preserved or improved. This is the first integration of a FV SW logic gate using a thin YIG film with device dimensions on the tens of micron scale, and it can be incorporated into further complex circuits because of the freedom in selecting the propagation direction of the FV SW. The improved footprint, wavelength, and isolation ratio were quantitatively compared to previous devices. Further development of device functionalities was analyzed using three dimensional calculations of SW propagation in the fabricated devices modeled on the experimental results.

\section{Results}

YIG preparation and characterization. All the YIG films used in this study were prepared on $10 \mathrm{~mm} \times 10 \mathrm{~mm} \times 0.5 \mathrm{~mm}$ substituted gadolinium gallium garnet (SGGG; $[\mathrm{GdCa}]_{3}[\mathrm{GaMgZr}]_{5} \mathrm{O}_{12}$ ) substrates with a (111) orientation using pulsed laser deposition (AOV PMAD-256). The temperature of the substrate during growth was approximately $850^{\circ} \mathrm{C}$ under an oxygen pressure of $2.6 \mathrm{~Pa}$ while the base pressure was $2 \times 10^{-4} \mathrm{~Pa}$. The repetition rate of the pulse laser was $15 \mathrm{~Hz}$, and the growth rate was $\sim 24 \mathrm{~nm} \mathrm{~min}^{-1}$. These values were similar to those used in our previous studies ${ }^{48,75}$.

The $2 \theta-\omega$ X-ray diffraction (XRD) patterns (Fig. 1a) were measured using a Rigaku Smartlab system with a $\mathrm{Cu}-\mathrm{K}(\alpha \mathrm{X}$-ray radiation source of wavelength $0.15418 \mathrm{~nm}$. The film was fully strained in plane, i.e. the in-plane lattice of the film was matched to that of SGGG substrate, as confirmed by reciprocal space mapping (RSM), Fig. 1c. The YIG unit cell is therefore rhombohedrally strained, and based on the out-of-plane lattice spacing of the (444) reflection and the in-plane lattice match with SGGG, we derived a unit cell side length $A$ of $1.2449 \mathrm{~nm}$ and corner angle of the unit cell $\theta_{\mathrm{CS}}$ of $90.337^{\circ}$. The unit cell volume of this rhombohedral lattice was $1.9628 \mathrm{~nm}^{3}$ $\left(=A^{3} \sqrt{1-3 \cos ^{2} \theta_{C S}+2 \cos 3 \theta_{C S}}\right)$. These values were similar to those used in our previous studies ${ }^{76}$. X-ray reflectometry (XRR) measurements showed that the films had a smooth surface with an average roughness, $R_{\mathrm{a}}$, of $0.47 \mathrm{~nm}$ and exhibited a density, $\rho$, of $5.17 \mathrm{~g} \mathrm{~cm}^{-3}$ (see Fig. 1b), and a thickness of $54 \mathrm{~nm}$. Composition measurements of a similar film confirmed the Y: Fe ratio was close to $3: 5$.

Measurements performed using a vibrating-sample magnetometer (VSM, Tamakawa TM-VSM261483HGC-SG) indicated that the films had an in-plane coercivity, $H_{c}$, of $0.37 \mathrm{Oe}$, a saturation magnetization, $M_{s}=143 \mathrm{emu} \mathrm{cm}^{-3}\left(4 \pi M_{\mathrm{s}}=1800 \mathrm{G}\right)$, and an out-of-plane saturation magnetic field obtained from a measurement of Faraday rotation angle loop, $H_{s}$, of $1.73 \mathrm{kOe}$.

The propagation of SWs in the film was characterized as described in a previous article ${ }^{76}$. Six pairs of antennas with various spacings were fabricated on the YIG film, and transmittance of signals between each pair of antennas was measured using a vector network analyzer (VNA). The measured intensity was plotted as a function of propagation length, yielding the attenuation length $L_{\text {att }}$ at which the SW intensity decayed by a factor $1 / \mathrm{e}$. The value of $L_{\text {att }}$ was converted to the net damping parameter $\alpha_{S W}$ which includes the extrinsic damping using ${ }^{1,76}$ 
(a)

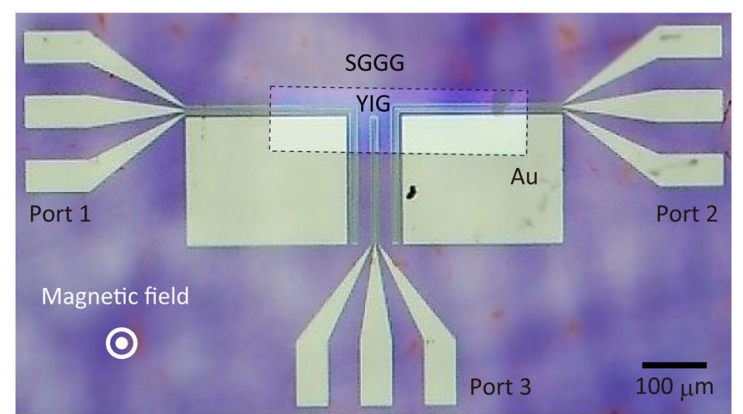

(b)

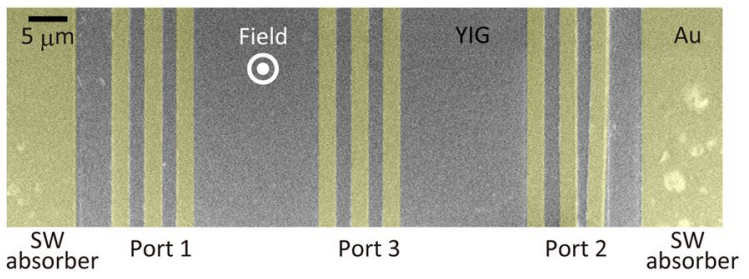

(c)

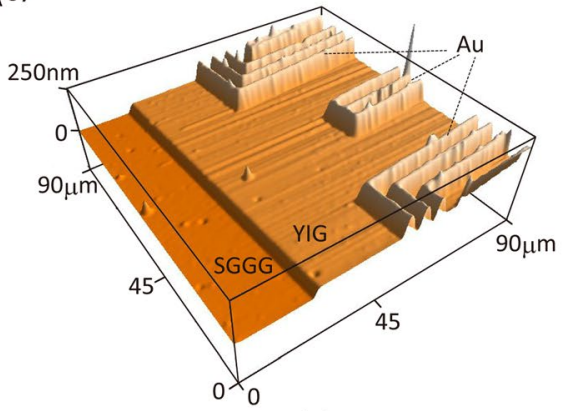

(d)

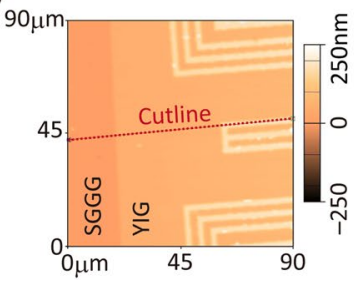

(e)

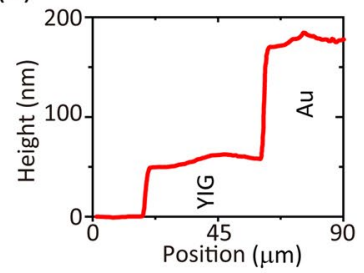

Figure 2. (a) Micrograph of FV SW interferometer fabricated using a $54 \mathrm{~nm}$ thick YIG waveguide. SW absorbers were fabricated at the edges of the waveguide. The YIG was magnetized out of plane. (b) SEM image of the interferometer. (c) AFM image of the interferometer. (d) Top view of the AFM image showing a cutline as a red dashed line. (e) Height profile along the cutline shown in (d).

$$
\alpha_{S W}=\frac{2\left(f_{0}^{2}-f^{2}\right)^{2}(1+\chi) d}{L_{a t t}\left(f_{0}^{2}+f^{2}\right) f \cdot f_{M}\left(\frac{2}{\chi}-k \cdot d\right)},
$$

where $\chi=f_{0} f_{M} /\left(f_{0}^{2}-f^{2}\right), f_{0}=-\gamma H_{e f f}, f_{M}=-\gamma \cdot 4 \pi M_{s}$, and $H_{\text {eff }}=H_{\text {appl }}+H_{A}^{\text {Dyn }}$. Here $f, k, d, \gamma, H_{\text {eff }}, H_{\text {appl }}$, and $H_{A}^{D y n}$ represent the frequency, the wavenumber, the YIG thickness, the gyromagnetic ratio $(=2.8 \mathrm{MHz} / \mathrm{Oe}$ for YIG), the effective magnetic field, the applied magnetic field, and the net magnetic anisotropy field obtained from spin wave spectroscopy (SWS, described in the following section). The measured damping factor of the YIG/SGGG for FV SWs was $\left(\alpha_{S W}=\sim 2^{4} \times 10^{-4}\right.$ at a frequency of $4 \mathrm{GHz}$ with $H_{a p p l}=2840 \mathrm{Oe}$, comparable to other reported values $\left(3.0 \times 10^{-362}, 8.79 \times 10^{-4664}, \times 10^{-460,64}, 2.8 \times 10^{-463}, 2.3 \times 10^{-465}, 8.0 \times 10^{-561}, 3.6 \times 10^{-549}\right.$,).

Spin wave device preparation. The YIG film was etched into a $400 \mu \mathrm{m} \times 100 \mu \mathrm{m}$ mesa. First, the YIG/ SGGG sample was cleaned using acetone, isopropyl alcohol (IPA), and deionized (DI) water with sonication, followed by baking at $180^{\circ} \mathrm{C}$ for $3 \mathrm{~min}$. Next, a $300 \mathrm{~nm}$ thick layer of a photoresist (ZEON, ZEP520A/ZEP-A =2: 1) was spin-coated at $2000 \mathrm{rpm}$ and baked at $180^{\circ} \mathrm{C}$ for $3 \mathrm{~min}$. An antistatic layer consisting of a conductive polymer (Showa Denko, ESPACER 300Z) was spin-coated at $2000 \mathrm{rpm}$ and baked at $60^{\circ} \mathrm{C}$ for $10 \mathrm{~min}$. The sample was then exposed using an electron beam system (EB, JEOL, JBX-6300FS) at a dose of $68 \mu \mathrm{C} \mathrm{cm}^{-2}$. Subsequently, the antistatic layer was removed using DI water. Next, the resist was developed (ZEON, ZED-N50) for $80 \mathrm{~s}$, and the sample was subsequently rinsed with IPA and baked at $120^{\circ} \mathrm{C}$ for $5 \mathrm{~min}$. A $\sim 50 \mathrm{~nm}$ thick $\mathrm{SiO}_{\mathrm{x}}$ mask was deposited on the sample by a RF ion-beam sputtering (IBS, OSI-WAVE RM17-0010) at a deposition rate of $\sim 2.6 \mathrm{~nm} \mathrm{~min}^{-1}$. The sample was then etched with phosphoric acid at $140^{\circ} \mathrm{C}$, with the etching process being controlled using an oil bath. The etching rate of the YIG film was $\sim 1.7 \mathrm{~nm} \mathrm{~s}^{-1}$. The remaining $\mathrm{SiO}_{\mathrm{x}}$ was removed by buffered hydrofluoric acid at an etching rate of $\sim 1.8 \mathrm{~nm} \mathrm{~s}^{-1}$.

On this YIG waveguide, three antennas (coplanar waveguides) composed of $90 \mathrm{~nm}$ thick $\mathrm{Au} / 10$-nm thick Ti layers were fabricated using the same resist and a liftoff process. The remaining Au/Ti was removed (ZEON, $Z D M A C)$ at $70^{\circ} \mathrm{C}$ for $20 \mathrm{~min}$ with $30 \mathrm{~s}$ sonication. The Ti layer served as adhesion for the Au layer. These films were deposited by direct current (DC) ion beam sputtering (TDY, 98012-RD). The fabricated $50 \mu \mathrm{m}$ wide contact pads and antenna patterns are shown in Fig. 2a. The scanning electron microscopy (SEM, JEOL, JEM-6700F) image in Fig. $2 \mathrm{~b}$ shows the SW injection port. The distance between the two edge signal lines was $\sim 53 \mu \mathrm{m}$, while the width and gap of each line in the antennas were $2.23 \mu \mathrm{m}$ and $1.92 \mu \mathrm{m}$, respectively. The antennas had a tilt of $1^{\circ}$ with respect to the edge of the YIG waveguide, and were shifted by $90 \mu \mathrm{m}$ from the edge, due to lithographic alignment errors. In addition, the ground line of the port 2 was slightly misaligned. However, we observed SW propagation and interference processes. The effects of this asymmetric and shifted structure are discussed below. The sample surface was also characterized by atomic force microscopy (AFM, Asylum Research, MFP-3D) as shown in Fig. 2c-e. The taper angle of the etched mesa YIG was $15^{\circ}$ with respect to the in-plane direction. The tip used in this observation (Olympus, OMCL-AC200TN-C3) had a radius of curvature of $30 \mathrm{~nm}$ (details of AFM conditions are shown in the Methods). Figure 2e showed that the height of the YIG part was between $49 \mathrm{~nm}$ and $62 \mathrm{~nm}$. These values show agreement with the result of XRR, $\sim 54 \mathrm{~nm}$. Their difference arises from overetching of the SGGG substrate by the phosphoric acid. Therefore, we assumed a $54 \mathrm{~nm}$ YIG thickness in this study. 
(a)

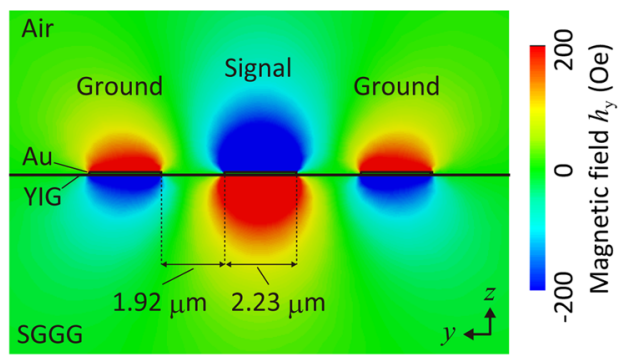

(b)

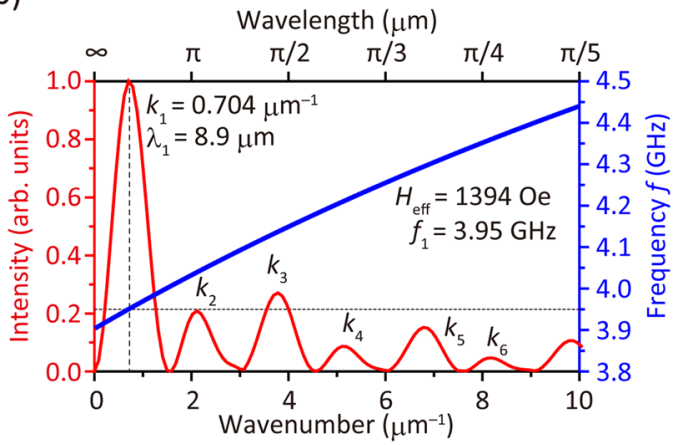

Figure 3. (a) Calculated RF magnetic field excited by the antenna used in the experiments. (b) Wavenumber spectra generated by the antenna (red line). The strongest peak exhibited wavenumber $k_{1}$ of $0.704 \mu \mathrm{m}^{-1}$, which corresponds to a wavelength $\lambda_{1}$ of $8.9 \mu \mathrm{m}$. Dispersion curve of FV SW at $H_{\text {eff }}=1394 \mathrm{Oe}$ is superposed (blue line), showing an excitation frequency $f_{1}$ of $3.95 \mathrm{GHz}$.

\begin{tabular}{|l|l|l|l|}
\hline Part & Antenna & Film & Substrate \\
\hline Material & $\mathrm{Au}$ & YIG & SGGG \\
\hline Thickness & $100 \mathrm{~nm}$ & $54 \mathrm{~nm}$ & $10 \mu \mathrm{m}$ \\
\hline Width & $2.23 \mu \mathrm{m}$ & $100 \mu \mathrm{m}$ & $300 \mu \mathrm{m}$ \\
\hline Gap & $1.92 \mu \mathrm{m}$ & - & - \\
\hline Conductivity, $\sigma$ & $4.561 \times 10^{7} \mathrm{Sm}^{-1}$ & - & - \\
\hline Permittivity, $\varepsilon_{\mathrm{r}}$ & - & 15.3 & 12.1 \\
\hline
\end{tabular}

Table 1. Material parameters used in antenna and SW simulations.

Calculation of spin wave wavelength. The wavelength of the SWs excited using these antennas was calculated using an RF simulator (CST Microwave Studio 2018) based on the finite integration technique ${ }^{77,78}$. This simulator, which employs Maxwell's equations, was based on three-dimensional (3D) objects similar to the experimental sample. The calculated RF magnetic field and the propagation of the SWs were in excellent agreement with the experimentally determined values ${ }^{79}$ within the dipolar $\mathrm{SW}^{1}$ region. This study treats the SWs as dipolar because $\lambda_{\mathrm{ex}} k^{2}$ «1, where $\lambda_{\mathrm{ex}}$ is the exchange constant $\left(3 \times 10^{-16} \mathrm{~m}^{2}\right.$ for YIG $\left.{ }^{1}\right)$. Hence, we can ignore the exchange term and use the RF simulator for calculating the SW. Figure 3 a shows the calculated EMW excited from the antenna modeled with the same dimensions as the sample used in the experiment. The material parameters used in this simulation are listed in Table 1. The titanium layer was not included in the calculation due to its low thickness.

The distribution of the $y$-component of the RF magnetic field, $h_{y}$ obtained at a frequency, $f$, of $3.95 \mathrm{GHz}$ at the bottom of an antenna with a width of $2.23 \mu \mathrm{m}$ and a gap of $1.92 \mu \mathrm{m}$ was fast-Fourier-transformed (FFT) into the wavenumber, $k^{65}$, as shown in Fig. 3b. The intensity was normalized. The wavelength of the strongest SW (the first mode), $\lambda_{1}$, was $8.9 \mu \mathrm{m}\left(k_{1}=0.704 \mu \mathrm{m}^{-1}\right)$. The dispersion curve of the SW was plotted in the same figure using ${ }^{1,79}$

$$
k=\frac{1}{d \sqrt{\mu}} \ln \frac{\sqrt{\mu}-1}{\sqrt{\mu}+1}
$$

where $\mu=1+f_{0} f_{M} /\left(f_{0}^{2}-f^{2}\right), f_{0}=-\gamma H_{e f f}$, and $f_{M}=-\gamma \cdot 4 \pi M_{s}$. Here $\gamma=2.8 \mathrm{MHz} / \mathrm{Oe}, H_{e f f}=1394 \mathrm{Oe}$, $4 \pi M_{s}=1800 \mathrm{G}$, and $d=54 \mathrm{~nm}$. This calculation determined the SW excitation frequency, wavelength, and the required magnetic field. $H_{\text {eff }}$ was determined as the best fit value that generated the FV SW with $\lambda_{1}=8.9 \mu \mathrm{m}$ at $f=3.95 \mathrm{GHz}$, in order to match the results of the experiments described below.

Spin wave spectroscopy. SW excitation was confirmed and the corresponding wavelengths were determined by SW spectroscopy (SWS) using a vector network analyzer (VNA, Agilent E5071C) and an electromagnet (Toei Scientific Industrial, TKSJ-V500-TYP), shown in Fig. 4. For SWS, three RF switches (Agilent, 3499B) connected the probes to the VNA. The intermediate frequency of the VNA was $100 \mathrm{~Hz}$, and the frequency was varied in steps of $3 \mathrm{MHz}$. A bias magnetic field was applied perpendicular to the YIG film using an electromagnet under proportional integral differential (PID) control with a Hall probe. The field was varied in steps of 20 Oe. The temperature of the sample was set to $35^{\circ} \mathrm{C}$ using a thermostat system (ATTS, A200). The three ports were placed in contact with RF probes (Cascade Microtech, SP-Z40-X-GSG-100) controlled by positioners (Cascade Microtech, RPP210-B-SP-AI).

Figure $5 \mathrm{a}, \mathrm{b}$ show the deembedded transmission from port 1 to port 3 and that from port 2 to port 3 . SW propagation is displayed as white lines (the broad horizontal line at $\sim 0.5 \mathrm{GHz}$ did not result from SWs). The white lines 


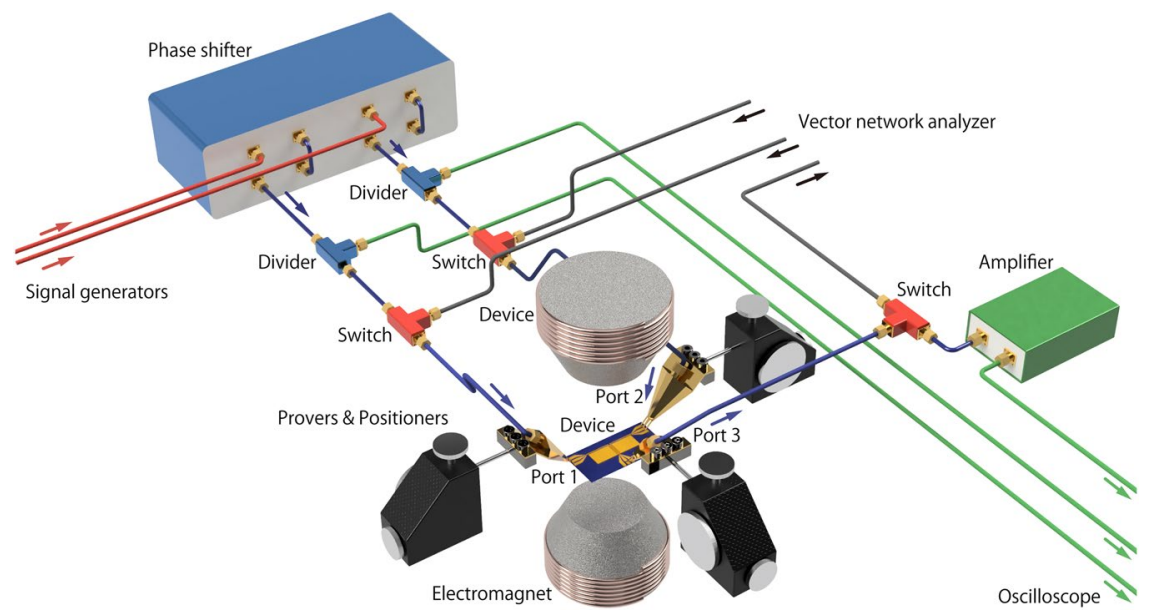

Figure 4. Experimental setup for SWS and SW interference. Three switches change the connection from the probes to the VNA or the oscilloscope.

appear discontinuous because of the 20 Oe step size of the applied field. To extract (de-embed) the transmitted SW, the spectrum at a magnetic field of 0.0 Oe was subtracted from the obtained spectra. This subtraction was conducted for the real part of the transmission from port 1 to port 3 and independently for the imaginary part, and the same was done for the transmission from port 2 to port 3. Examples are shown in Fig. 5c,d. The largest peak corresponds to the first-order SW $\left(\lambda_{1}\right.$ of $\left.8.9 \mu \mathrm{m}\right)$ and was obtained at $f=3.95 \mathrm{GHz}$ with $H_{\text {appl }}=2800$ Oe. The positions of the spectral peaks corresponding to $\lambda_{1}$ in Fig. 5c,d were similar, with the difference being $3 \mathrm{MHz}$. Thus, their coherence is considered high enough for interference. The peak transmission at $H_{\text {appl }}=2.8 \mathrm{kOe}$ is only $\sim 1.7$ times larger than that at $H_{\text {appl }}=0.0 \mathrm{kOe}$, which is attributed to the low conversion efficiency of the SW excitation antennas. This could be increased by the use of meander antennas. In Fig. 5a, the intersection of the measured white line and the horizontal axis shows the net magnetic anisotropy field $H_{A}^{D y n}=-1346 \mathrm{Oe}$, the same as in Fig. 5b. Thus, the effective magnetic field $H_{\text {eff }}=H_{\text {appl }}+H_{A}^{D y n}=2800-1346=1454$ Oe at $f=3.95 \mathrm{GHz}$. This value of $H_{\text {eff }}$ is 60 Oe larger than that used in the calculation of the dispersion curve shown in Fig. 3b, 1394 Oe. This modest disagreement might be caused by the difference between the ideal models ${ }^{1}$ used in the calculation and the actual sample structure, including thickness variations in YIG observed in the AFM image (Fig. 2e) and/ or inhomogeneity of magnetic properties along the thickness direction especially near the boundary between the YIG and the substrate ${ }^{47,49}$.

Spin wave interference. The two input ports (ports 1 and 2) were connected to the same probe system as that used in the SWS system shown in Fig. 4 and were switched using an RF switch without reconnecting any cables. These input lines were connected to the individual signal generators (Agilent E8257C and HP 83732 A) through power dividers (HP 11636B) and a two-port phase shifter (Colby Instruments PLD-200A-625DS) in order to be able to adjust the difference in the injection phase independently. The divided lines were connected to a digital oscilloscope (Tektronix DSA70804) to monitor the input signals in situ. The output port (port 3) was connected to the oscilloscope after amplifying the signal by $30 \mathrm{~dB}$ (Amplifier Research, 1S1G4A). All the input voltages were adjusted independently, so that each input voltage was $350 \mathrm{mV}$. The two signal generators were synchronized using a $10 \mathrm{MHz}$ signal generated by the HP $8647 \mathrm{~A}$ generator. The spectral difference between the two inputs was less than $10 \mathrm{~Hz}$.

Figure 6 shows the interference results of the FV SWs at $f=3.95 \mathrm{GHz}$ for $H_{\text {appl }}=2.80 \mathrm{kOe}$. The interference of the SWs changed as a function of the phase difference, $\xi$, between ports 1 and 2 . This change was analyzed using a fitting technique. The output synthesized wave, $V$, can be expressed as $V=A \sin \omega t+B \sin (\omega t+\xi)$, where $A$ and $B$ are the amplitudes of the input SWs, $\omega$ is the angular frequency, and $t$ is the time. This was converted to

$$
V=\sqrt{2} A \sqrt{1+\cos \xi} \sin (\omega t+\Delta),
$$

where $\Delta=\cos ^{-1} \sqrt{(1+\cos \xi) / 2}$ in the case of $A=B$. For the analysis the amplitudes were assumed to be the same for simplicity, even though their amplitudes had $\sim 10 \%$ difference as shown in Fig. $5 c$,d. Therefore, the peak-to-peak voltage was

$$
V_{p p}=2 \sqrt{2} A \sqrt{(1+\cos \xi)} .
$$

However, a bias voltage of $1 \mathrm{mV}$ was added as a fitting factor to Eq. (4), and $A$ was set to $2.2 \mathrm{mV}$. Overall, this theoretical line was in good agreement with the experimental results, confirming that constructive and destructive SW interference had indeed occurred.

A phase difference of $0^{\circ}$ corresponds to the two input signals (" 1 ", "1") or ("0", " 0 "). In this case, the output $V_{p p}$ is higher than a threshold voltage (e.g. $5 \mathrm{~V}$ ), and corresponds to an output signal of " 1 ". In contrast, a phase 
(a)

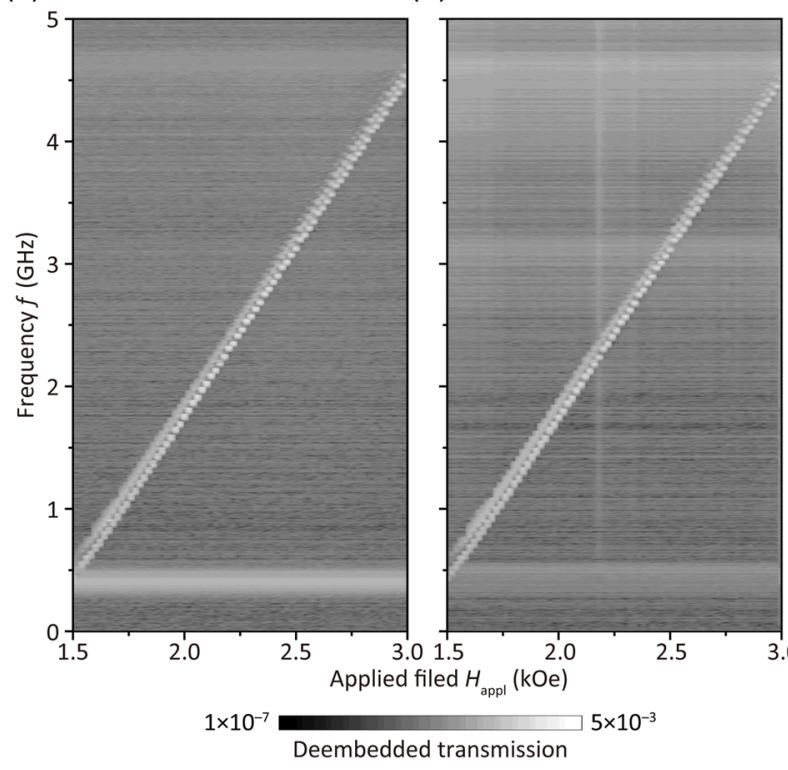

(c)

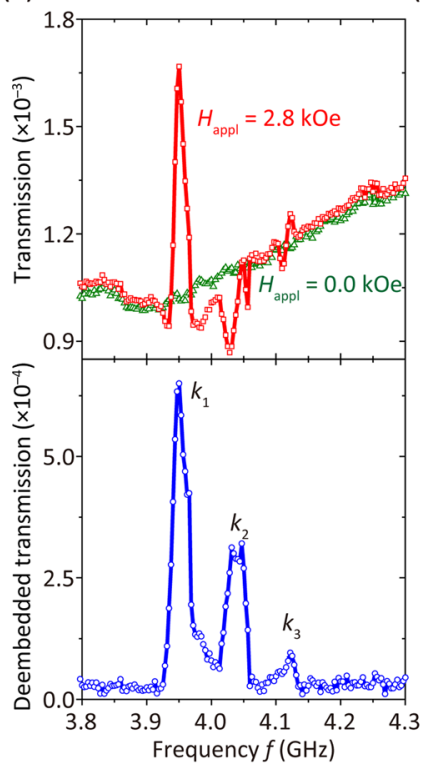

(d)

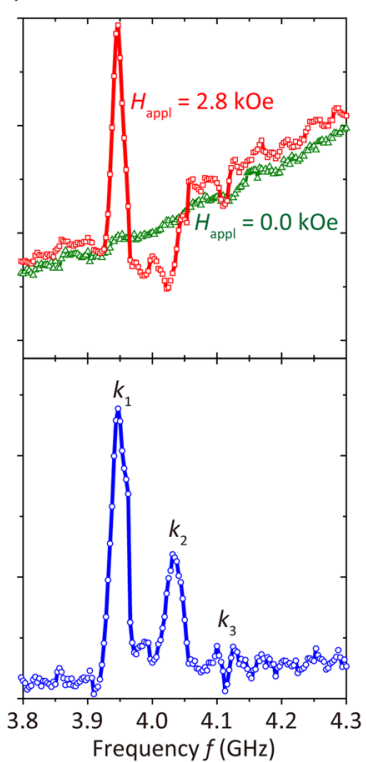

Figure 5. (a,b) SWS of deembedded transmission from ports 1-3 and ports 2-3, respectively. (c,d) Transmission from ports 1-3 and ports 2-3 versus frequency, respectively. Red squares and green triangles show data at an applied magnetic field, $H_{\text {appl }}$, of $2.8 \mathrm{kOe}$ and $0.0 \mathrm{kOe}$, respectively. Blue circles show deembedded transmission spectra.

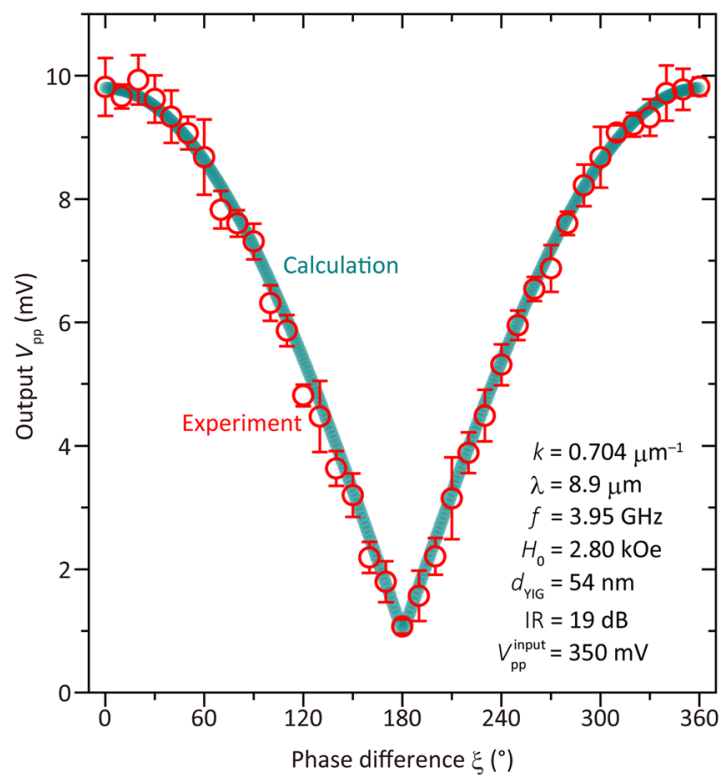

Figure 6. FV SW interference using $54 \mathrm{~nm}$ thick YIG waveguide. Red circles show experimental results. Error bars show standard deviation $1 \sigma$ for five duplicated measurement. Green bold line shows calculated values using Eq. (4).

difference of $180^{\circ}$ corresponds to input signals of ("0", "1") or (" 1 ", " 0 "). In this case, the output $V_{p p}$ is lower than a threshold voltage. This corresponds to an output signal of " 0 ". This behavior represents an exclusive NOR (XNOR) logic gate, expressing the combination of $\mathrm{EXOR}^{80}$ and NOT gate.

The maximum and minimum output voltages were $9.80 \mathrm{mV}$ and $1.06 \mathrm{mV}$, respectively. Thus, the isolation ratio (IR), which measures the on/off performance of the device and is defined as 20 times the log of the ratio of maximum to minimum output voltage, was $I R=19 \mathrm{~dB}$. This was $5 \mathrm{~dB}$ larger than that obtained in a previous study of SW interference based on an $18 \mu \mathrm{m}$ thick YIG waveguide ${ }^{11}$. Moreover, this IR was obtained in a device with a footprint (i.e., the distance between the two edge electrodes) that was $4 \times 10^{4}$ times smaller than that of ref. ${ }^{11}$, at $\sim 4 \times 10^{-3} \mathrm{~mm}^{2}$. This was possible because of the shortening of the wavelength of the SWs due to the antenna 


\begin{tabular}{|l|l|l|l|}
\hline & $\begin{array}{l}\text { 54-nm-thick } \\
\text { YIG (this study) }\end{array}$ & $\begin{array}{l}\mathbf{1 8 - \mu m - t h i c k} \\
\text { YIG }^{11}\end{array}$ & $\begin{array}{l}\text { 35-nm-thick } \\
\text { permalloy }^{13}\end{array}$ \\
\hline SW wavelength, $\lambda$ & $8.9 \mu \mathrm{m}$ & $780 \mu \mathrm{m}$ & $16 \mu \mathrm{m}$ \\
\hline SW wavenumber, $k$ & $0.704 \mu \mathrm{m}^{-1}$ & $8.06 \mu \mathrm{m}^{-1}$ & $0.4 \mu \mathrm{m}^{-1}$ \\
\hline Footprint & $4 \times 10^{-3} \mathrm{~mm}^{2}$ & $1 \times 10^{1} \mathrm{~mm}^{2}$ & $4 \times 10^{-3} \mathrm{~mm}^{2}$ \\
\hline Distance between input ports & $53 \mu \mathrm{m}$ & $10 \mathrm{~mm}$ & $60 \mu \mathrm{m}$ \\
\hline Input voltage & $350 \mathrm{mV}$ & $200 \mathrm{mV}$ & $490 \mathrm{mV}$ \\
\hline Maximum output voltage & $9.80 \mathrm{mV}$ & $6.31 \mathrm{mV}$ & $0.37 \mathrm{mV}$ \\
\hline Minimum output voltage & $1.06 \mathrm{mV}$ & $1.30 \mathrm{mV}$ & $0.03 \mathrm{mV}$ \\
\hline Isolation ratio, IR & $19 \mathrm{~dB}$ & $14 \mathrm{~dB}$ & $22 \mathrm{~dB}$ \\
\hline Applied field, $H_{0}$ & $2.80 \mathrm{kOe}$ & $3.05 \mathrm{kOe}$ & $0.44 \mathrm{kOe}$ \\
\hline Frequency, $f$ & $3.95 \mathrm{GHz}$ & $4.0 \mathrm{GHz}$ & $6.5 \mathrm{GHz}$ \\
\hline SW mode & Forward volume & Forward volume & Surface \\
\hline
\end{tabular}

Table 2. SW device performance observed in this study and those reported in previous works ${ }^{11,13}$.

(a)

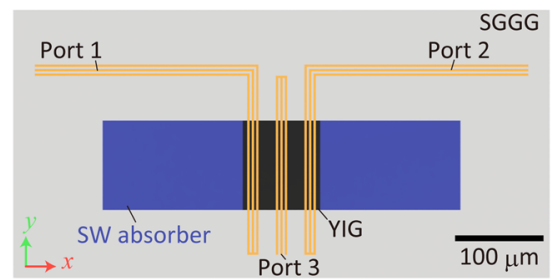

(b)

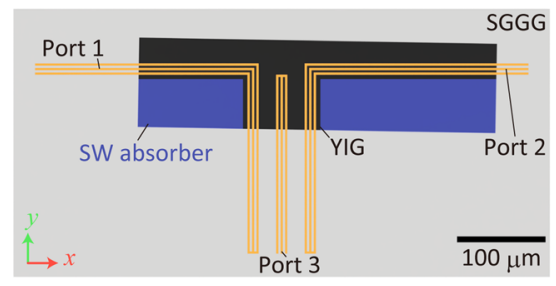

(c)

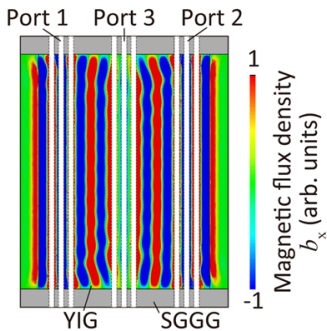

(d)

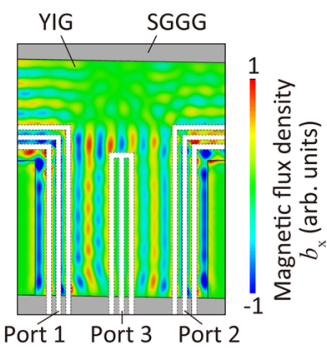

(e)

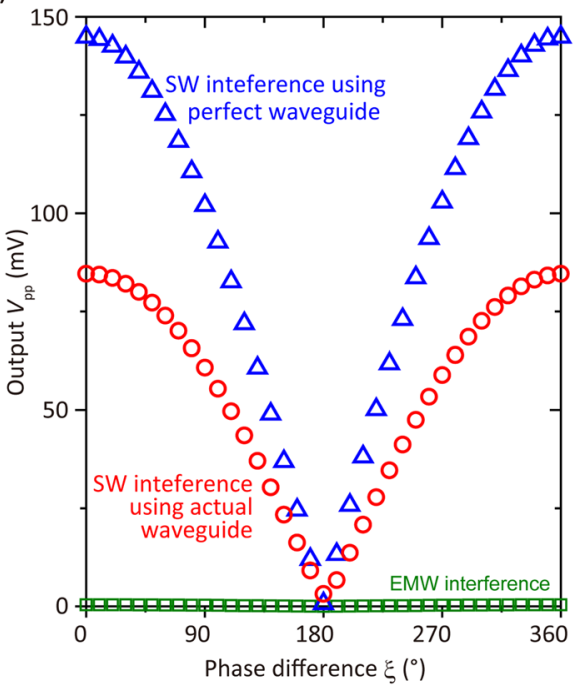

Figure 7. (a) 3D model of the interferometer with perfect alignment. (b) Model of the actual interferometer with $1^{\circ}$ tilt and $90 \mu \mathrm{m}$ shift between the waveguide and the antenna. (c,d) The distribution of magnetic flux density $b_{\mathrm{x}}$ in the vicinity of the output port at the center plane in the thickness direction of YIG in the case of (c) perfect waveguide and (d) actual waveguide. Phase difference was zero. (e) Simulated interference results for perfect waveguide (blue triangles) and actual device (red circles). An asymmetric wave interference state resulted in imperfect destructive interference. These results were all calculated at $f=3.95 \mathrm{GHz}$ to see $\mathrm{FV}$ SW interference, but the calculation of EMW interference (green squares) was conducted at $f=3.80 \mathrm{GHz}$ to eliminate the contribution of SWs.

design, and the integration of the antennas on the single-crystalline YIG film. The device performance parameters are listed in Table 2; the results of previous studies are also shown for comparison. The footprint of the XNOR gate, four orders of magnitude smaller than that in ref. ${ }^{11}$, represents an obvious advantage of the integration. Further, the output voltage was an order of magnitude larger than that in the case of permalloy because of the low losses. In addition, IR was no lower than that in the case of bulk YIG, indicating that the fabrication of the SW device did not adversely affect the characteristics of the SW propagation.

Simulation of spin wave interference. Although interference was demonstrated successfully in this study, imperfect destructive interference was observed. The remaining voltage in the out-of-phase state was $1 \mathrm{mV}$. To investigate the cause of this, a finite integration technique simulation was performed on a 3D model of the sample structure, including the tilt angle of $1^{\circ}$ and a parallel micron-scale shifting of the antennas on the YIG waveguide, as shown in Fig. 7a,b. The material parameters used were shown in Table 1. SW absorbers were introduced into the model in the areas with high damping $\left(\alpha=1 \times 10^{-2}\right)$. The damping factor of YIG was taken to be $2.4 \times 10^{-4}$, which is the same as the experimental value. All ports had an impedance of $50 \Omega$, and were set as the discrete port in the software. A simulation corresponding to the ideal situation, wherein the sample had a completely symmetrical structure, was also performed for comparison, as shown in Fig. 7a. 


\begin{tabular}{|l|l|}
\hline & Name/Parameter \\
\hline Model & Aslyum Research, MFP-3D \\
\hline Software version & 14.30 .157 worked with Igor Pro ver. 6.3.7.2. \\
\hline Scanning area (maximum) & $90 \mu \mathrm{m} \times 90 \mu \mathrm{m}$ \\
\hline Scanning rate & 0.2496 \\
\hline Scanning line & 256 \\
\hline Imaging mode & Contact \\
\hline Spring constant & $0.03311 \mathrm{~N} / \mathrm{m}$ \\
\hline AmpinvOLS & $339.86 \mathrm{~nm} / \mathrm{V}$ \\
\hline Defl. InvOLS & $311.8 \mathrm{~nm} / \mathrm{V}$ \\
\hline Integral gain & 1.2 \\
\hline Proportional gain & 1 \\
\hline Drive Frequency & $11.478 \mathrm{kHz}$ \\
\hline Q value & 34.9 \\
\hline Resonance Frequency & $11.54 \mathrm{kHz}$ \\
\hline
\end{tabular}

Table 3. Specification and setting of the AFM. AmpinvOLS and Defl. InvOLS show the amplitude inverse optical lever sensitivity, and the deflection inverse optical lever sensitivity, respectively.

\begin{tabular}{|l|l|}
\hline & Name/Parameter \\
\hline Model & Olympus, OMCL-TR400PB-1, series B \\
\hline Position offset & $-4 \mu \mathrm{m}$ \\
\hline Shape & Pyramidal \\
\hline Tip height & $2.9 \mu \mathrm{m}$ \\
\hline Tip curvature radius & $30 \mathrm{~nm}$ \\
\hline Tip material & $\mathrm{SiN}$ \\
\hline Lever material & $\mathrm{SiN}$ \\
\hline Coating material (both side) & $\mathrm{Au}$ \\
\hline
\end{tabular}

Table 4. Specification of the AFM probe.

The results indicated that the asymmetrical interferometer exhibited a larger bias voltage than did the symmetrical one as shown in Fig. $7 \mathrm{~d}$. In the case of the symmetric structure, cancellation of the SWs was observed at the center of port 3 (see Fig. 7c). Hence, the output voltage was almost zero, as can be seen from Fig. 7e. In addition, the voltage corresponding to the in-phase state was lower than that for the symmetrical case. This was because of the decrease in the overlapping length of the antenna on the YIG waveguide. In the actual waveguide, only $\sim 62.4 \%$ of the antenna covered the YIG waveguide, and the output voltage was approximately $61.9 \%$ of that for the ideal case, indicating a quantitative agreement between the two. Obviously, these issues can be prevented by ensuring precise alignment between the antenna layer and the YIG waveguide layer. Therefore, such an improvement should increase both the IR and the robustness of operation of the XNOR logic gate. Supplementally, the interference of EMW was calculated at $f=3.80 \mathrm{GHz}$ where SWs cannot be excited. This result shows the output $V_{p p}$ generated by EMW was three orders of magnitude lower than that of SWs. This is negligible and it can be concluded that the interference of FV SWs was demonstrated successfully in this experiment.

\section{Conclusion}

In this study, a three-port XNOR gate based on FV SW interference at a frequency of $\sim 3.95 \mathrm{GHz}$ was demonstrated, using a $54 \mathrm{~nm}$ thick single crystalline YIG waveguide grown on an SGGG substrate and three coplanar waveguides exciting FV SWs with a wavelength of $8.9 \mu \mathrm{m}$. The device footprint was $4 \times 10^{-4}$ times that reported previously ${ }^{11}$, with the device showing a $19 \mathrm{~dB}$ isolation ratio. The integration of the $\mathrm{SW}$ device did not adversely affect the properties of the SWs, which is attributed to the high quality of the fabricated YIG film. The geometry of the SW waveguide was analyzed, and it was concluded that the asymmetric structure arising from fabrication was responsible for the imperfect destructive interference observed. The proposed technique should find use in the development of fundamental logic gates based on thin YIG films. Thus, this study is an important milestone towards the realization of more complex functionality in SW ICs.

\section{Methods}

Deposition of $\mathrm{SiO}_{\mathbf{x}}$. The $\mathrm{SiO}_{\mathrm{x}}$ was coated by RF ion beam sputtering (IBS, OSI-WAVE RM17-0010) at room temperature using a sintered 4 inch $\mathrm{SiO}_{\mathrm{x}}$ target in $10 \mathrm{mTorr}(=1.3 \mathrm{~Pa})$ and $7.5 \mathrm{sccm}$ Ar gas flow and $6.0 \mathrm{sccm} \mathrm{O}_{2}$ gas flow with an RF power of $114 \mathrm{~W}$. The base pressure was $6 \times 10^{-6} \operatorname{Torr}\left(=8 \times 10^{-4} \mathrm{~Pa}\right)$.

Deposition of Au and Ti. A $90 \mathrm{~nm}$ thick $\mathrm{Au}$ and a $10 \mathrm{~nm}$ thick Ti were deposited by DC IBS (TDY, 98012-RD) at room temperature using a 4 inch $\mathrm{Au}$ and Ti target. Au was deposited in 3 mTorr $(=0.4 \mathrm{~Pa})$ pressure 
and $5.0 \mathrm{sccm}$ Ar gas flow with a DC power of $40 \mathrm{~W}$ at a deposition rate of $16 \mathrm{~nm} \mathrm{~min}^{-1}$. Ti was deposited in 20 mTorr $(=2.7 \mathrm{~Pa})$ pressure and $5.0 \mathrm{sccm}$ Ar gas flow with $50 \mathrm{~W}$ power. The deposition rate was $10 \mathrm{~nm} \mathrm{~min}^{-1}$.

AFM. Specifications and settings of the AFM and its probe used in the experiments were shown in Table 3 and Table 4.

Received: 18 July 2019; Accepted: 22 October 2019;

Published online: 11 November 2019

\section{References}

1. Stancil, D. D. \& Prabhakar, A. Spin Waves. (Springer, New York, 2009).

2. Stancil, D. D. Theory of Magnetostatic Waves. (Springer, New York, 1993).

3. Demokritov, S. O. \& Slavin, A. N. Magnonics. (Springer, New York, 2012).

4. Yu, H., Xiao, J. \& Pirro, P. Magnon Spintronics. J. Magn. Magn. Mater. 450, 1-2 (2018)

5. Chumak, A. V., Vasyuchka, V. I., Serga, A. A. \& Hillebrands, B. Magnon spintronics. Nat. Phys. 11, 453-461 (2015).

6. Kruglyak, V. V., Demokritov, S. O. \& Grundler, D. Magnonics. J. Phys. D: Appl. Phys. 43, 264001 (2010).

7. Schneider, T. et al. Realization of spin-wave logic gates. Appl. Phys. Lett. 92, 022505 (2008).

8. Sato, N., Lee, S.-W., Lee, K.-J. \& Sekiguchi, K. Current-induced modulation of backward spin-waves in metallic microstructures. J. Phys. D: Appl. Phys. 50, 094004 (2017).

9. Kostylev, M. P., Serga, A. A., Schneider, T., Leven, B. \& Hillebrands, B. Spin-wave logical gates. Appl. Phys. Lett. 87, 153501 (2005).

10. Pirro, P. et al. Interference of coherent spin waves in micron-sized ferromagnetic waveguides. Phys. Status Solidi B 248, 2404-2408 (2011).

11. Kanazawa, N. et al. Demonstration of a robust magnonic spin wave interferometer. Sci. Rep. 6, 30268 (2016).

12. Serga, A. A., Chumak, A. V. \& Hillebrands, B. YIG magnonics. J. Phys. D: Appl. Phys. 43, 264002 (2010).

13. Sato, N., Sekiguchi, K. \& Nozaki, Y. Electrical demonstration of spin-wave logic operation. Appl. Phys. Express 6, 063001 (2013).

14. Kanazawa, N. et al. The role of Snell's law for a magnonic majority gate. Sci. Rep. 7, 7898 (2017).

15. Fischer, T. et al. Experimental prototype of a spin-wave majority gate. Appl. Phys. Lett. 110, 152401 (2017).

16. Krawczyk, M. \& Grundler, D. Review and prospects of magnonic crystals and devices with reprogrammable band structure. J. Phys.: Condens. Matter 26, 123202 (2014).

17. Chumak, A. V., Serga, A. A. \& Hillebrands, B. Magnonic crystals for data processing. J. Phys. D: Appl. Phys. 50, 244001 (2017).

18. Kanazawa, N., Goto, T. \& Inoue, M. Spin wave localization in one-dimensional magnonic microcavity comprising yttrium iron garnet. J. Appl. Phys. 116, 083903 (2014).

19. Kanazawa, N. et al. Metal thickness dependence on spin wave propagation in magnonic crystal using yttrium iron garnet. J. Appl. Phys. 117, 17E510 (2015).

20. Sykes, C. G., Adam, J. D. \& Collins, J. H. Magnetostatic wave propagation in a periodic structure. Appl. Phys. Lett. 29, 388-391 (1976).

21. Nikitov, S. A., Tailhades, P. \& Tsai, C. S. Spin waves in periodic magnetic structures-magnonic crystals. J. Magn. Magn. Mater. 236, $320-330$ (2001).

22. Kuchko, A. N., Sokolovskii, M. L. \& Kruglyak, V. V. Spin wave spectrum of a magnonic crystal with an internally structured defect. Physica B: Condensed Matter 370, 73-77 (2005).

23. Kruglyak, V. V., Sokolovskii, M. L., Tkachenko, V. S. \& Kuchko, A. N. Spin-wave spectrum of a magnonic crystal with an isolated defect. J. Appl. Phys. 99, 08C906 (2006).

24. Chumak, A. V., Serga, A. A., Hillebrands, B. \& Kostylev, M. P. Scattering of backward spin waves in a one-dimensional magnonic crystal. Appl. Phys. Lett. 93, 022508 (2008).

25. Chumak, A. V. et al. Spin-wave propagation in a microstructured magnonic crystal. Appl. Phys. Lett. 95, 262508 (2009).

26. Chumak, A. V., Neumann, T., Serga, A. A., Hillebrands, B. \& Kostylev, M. P. A current-controlled, dynamic magnonic crystal. J. Phys. D: Appl. Phys. 42, 205005 (2009).

27. Wang, Z. K. et al. Observation of frequency band gaps in a one-dimensional nanostructured magnonic crystal. Appl. Phys. Lett. 94, $083112(2009)$.

28. Chumak, A. V., Serga, A. A., Wolff, S., Hillebrands, B. \& Kostylev, M. P. Scattering of surface and volume spin waves in a magnonic crystal. Appl. Phys. Lett. 94, 172511 (2009).

29. Kim, S.-K., Lee, K.-S. \& Han, D.-S. A gigahertz-range spin-wave filter composed of width-modulated nanostrip magnonic-crystal waveguides. Appl. Phys. Lett. 95, 082507 (2009).

30. Chumak, A. V. et al. All-linear time reversal by a dynamic artificial crystal. Nat. Comm. 1, 141 (2010).

31. Chi, K. H., Zhu, Y., Mao, R. W., Dolas, J. P. \& Tsai, C. S. An approach for analysis of magnetostatic volume waves in magnonic crystals. J. Appl. Phys. 109, 07D320 (2011).

32. Karenowska, A. D., Chumak, A. V., Serga, A. A., Gregg, J. F. \& Hillebrands, B. Employing magnonic crystals to dictate the characteristics of auto-oscillatory spin-wave systems. J. Phys.: Conf. Ser. 303, 012007 (2011).

33. Ma, F. S., Lim, H. S., Zhang, V. L., Ng, S. C. \& Kuok, M. H. Magnonic band structure investigation of one-dimensional bi-component magnonic crystal waveguides. Nanoscale Research Letters 7, 498 (2012).

34. Chen, C. H., Qiu, R. Z., Chang, C. H. \& Hsueh, W. J. Strongly localized modes in one-dimensional defect-free magnonic quasicrystals. AIP Advances 4, 087102 (2014).

35. Sheshukova, S. E., Beginin, E. N., Sadovnikov, A. V., Sharaevsky, Y. P. \& Nikitov, S. A. Multimode propagation of magnetostatic waves in a width-modulated yttrium-iron-garnet waveguide. IEEE Magnetics Letters 5, 1-4 (2014).

36. Morozova, M. A. et al. Band gap control in a line-defect magnonic crystal waveguide. Appl. Phys. Lett. 107, 242402 (2015).

37. Bessonov, V. D. et al. Magnonic band gaps in YIG-based one-dimensional magnonic crystals: An array of grooves versus an array of metallic stripes. Phys. Rev. B 91, 104421 (2015).

38. Ordóñez-Romero, C. L. et al. Mapping of spin wave propagation in a one-dimensional magnonic crystal. J. Appl. Phys. 120, 043901 (2016).

39. Sadovnikov, A. V. et al. Nonlinear spin wave coupling in adjacent magnonic crystals. Appl. Phys. Lett. 109, 042407 (2016).

40. Vogel, M. et al. Optically reconfigurable magnetic materials. Nat Phys 11, 487-491 (2015).

41. Sebastian, T. et al. Low-damping spin-wave propagation in a micro-structured Co2Mn0.6Fe0.4Si Heusler waveguide. Appl. Phys. Lett. 100, 112402 (2012).

42. Kubota, T. et al. Half-metallicity and Gilbert damping constant in Co2FexMn1-xSi Heusler alloys depending on the film composition. Appl. Phys. Lett. 94, 122504 (2009).

43. Kobayashi, K. et al. Damping constants for permalloy single-crystal thin films. IEEE Trans. Magn. 45, 2541-2544 (2009).

44. Kalarickal, S. S. et al. Ferromagnetic resonance linewidth in metallic thin films: Comparison of measurement methods. J. Appl. Phys. 99, 093909 (2006).

45. Vlaminck, V. \& Bailleul, M. Current-Induced Spin-Wave Doppler Shift. Science 322, 410-413 (2008). 
46. Bilzer, C. et al. Study of the dynamic magnetic properties of soft CoFeB films. J. Appl. Phys. 100, 053903 (2006).

47. Fu, J. et al. Epitaxial growth of $\mathrm{Y}_{3} \mathrm{Fe}_{5} \mathrm{O}_{12}$ thin films with perpendicular magnetic anisotropy. Appl. Phys. Lett. 110, 202403 (2017).

48. Onbasli, M. C. et al. Pulsed laser deposition of epitaxial yttrium iron garnet films with low Gilbert damping and bulk-like magnetization. APL Materials 2, 106102 (2014).

49. Lutsev, L. V. et al. Low-relaxation spin waves in laser-molecular-beam epitaxy grown nanosized yttrium iron garnet films. Appl. Phys. Lett. 108, $182402(2016)$.

50. Li, S. et al. Epitaxial patterning of nanometer-thick $\mathrm{Y}_{3} \mathrm{Fe}_{5} \mathrm{O}_{12}$ films with low magnetic damping. Nanoscale 8, 388-394 (2016).

51. Tang, C. et al. Exquisite growth control and magnetic properties of yttrium iron garnet thin films. Appl. Phys. Lett. 108, 102403 (2016).

52. Hauser, C. et al. Yttrium iron garnet thin films with very low damping obtained by recrystallization of amorphous material. Sci. Rep. 6, 20827 (2016)

53. Kahl, S. \& Grishin, A. M. Pulsed laser deposition of $\mathrm{Y}_{3} \mathrm{Fe}_{5} \mathrm{O}_{12}$ and $\mathrm{Bi}_{3} \mathrm{Fe}_{5} \mathrm{O}_{12}$ films on garnet substrates. J. Appl. Phys. 93, 6945-6947 (2003).

54. Kubota, M. et al. Systematic control of stress-induced anisotropy in pseudomorphic iron garnet thin films. J. Magn. Magn. Mater. 339, 63-70 (2013).

55. Tepper, T. \& Ross, C. A. Pulsed laser deposition of iron oxide films. J. Appl. Phys. 91, 4453-4456 (2002).

56. Levy, M., Chakravarty, A., Huang, H.-C. \& Osgood, R. M. Jr. Large magneto-optic enhancement in ultra-thin liquid-phase-epitaxy iron garnet films. Appl. Phys. Lett. 107, 011104 (2015).

57. Chang, H. et al. Nanometer-Thick Yttrium Iron Garnet Films With Extremely Low Damping. IEEE Magnetics Letters 5, 1-4 (2014).

58. Liu, T. et al. Ferromagnetic resonance of sputtered yttrium iron garnet nanometer films. J. Appl. Phys. 115, 17A501 (2014).

59. Maendl, S., Stasinopoulos, I. \& Grundler, D. Spin waves with large decay length and few $100 \mathrm{~nm}$ wavelengths in thin yttrium iron garnet grown at the wafer scale. Appl. Phys. Lett. 111, 012403 (2017).

60. Collet, M. et al. Spin-wave propagation in ultra-thin YIG based waveguides. Appl. Phys. Lett. 110, 092408 (2017)

61. Liu, C. et al. Long-distance propagation of short-wavelength spin waves. Nat. Comm. 9, 738 (2018)

62. Balinskiy, M. et al. Spin wave excitation in sub-micrometer thick $\mathrm{Y}_{3} \mathrm{Fe}_{5} \mathrm{O}_{12}$ films fabricated by pulsed laser deposition on garnet and silicon substrates: A comparative study. J. Appl. Phys. 122, 123904 (2017).

63. Pirro, P. et al. Spin-wave excitation and propagation in microstructured waveguides of yttrium iron garnet/Pt bilayers. Appl. Phys. Lett. 104, 012402 (2014).

64. Hahn, C. et al. Measurement of the intrinsic damping constant in individual nanodisks of $\mathrm{Y}_{3} \mathrm{Fe}_{5} \mathrm{O}_{12}$ and $\mathrm{Y}_{3} \mathrm{Fe}_{5} \mathrm{O}_{12} \mid \mathrm{Pt}$. Appl. Phys. Lett. 104, 152410 (2014).

65. Yu, H. et al. Magnetic thin-film insulator with ultra-low spin wave damping for coherent nanomagnonics. Sci. Rep. 4, 6848 (2014)

66. Jungfleisch, M. B. et al. Spin waves in micro-structured yttrium iron garnet nanometer-thick films. J. Appl. Phys. 117, 17D128 (2015),

67. Chen, J. et al. Spin wave propagation in perpendicularly magnetized nm-thick yttrium iron garnet films. J. Magn. Magn. Mater. 450, 3-6 (2018).

68. Okamura, Y., Ishida, M. \& Yamamoto, S. Magnetooptic rib waveguides in YIG: an experiment. Appl. Opt. 23, 124-126 (1984).

69. Zhu, N. et al. Patterned growth of crystalline $\mathrm{Y}_{3} \mathrm{Fe}_{5} \mathrm{O}_{12}$ nanostructures with engineered magnetic shape anisotropy. Appl. Phys. Lett. 110,252401 (2017).

70. Krysztofik, A. et al. Ultra-low damping in lift-off structured yttrium iron garnet thin films. Appl. Phys. Lett. 111, 192404 (2017).

71. Ustinov, A. B., Kalinikos, B. A. \& Lahderanta, E. Nonlinear phase shifters based on forward volume spin waves. J. Appl. Phys. 113, 113904 (2013).

72. Adam, J. D. YIG film characterization for MSW devices. Circ. Syst. Signal Process. 4, 105-113 (1985).

73. Talisa, S., Emtage, P., Daniel, M. \& Adam, J. Passband ripple observed in MSFVW delay lines. IEEE Trans. Magn. 22, 856-858 (1986).

74. Goto, T. et al. Spin wave differential circuit for realization of thermally stable magnonic sensors. Appl. Phys. Lett. 106, 132412 (2015).

75. Kehlberger, A. et al. Enhanced magneto-optic Kerr effect and magnetic properties of $\mathrm{CeY}_{2} \mathrm{Fe}_{5} \mathrm{O}_{12}$ epitaxial thin films. Phys. Rev. Applied 4, 014008 (2015).

76. Yoshimoto, T. et al. Static and dynamic magnetic properties of single-crystalline yttrium iron garnet films epitaxially grown on three garnet substrates. Adv. Electron. Mater. 4, 1800106 (2018).

77. Weiland, T., Timm, M. \& Munteanu, I. A practical guide to 3-D simulation. IEEE Microwave Magazine 9, 62-75 (2008).

78. Weiland, T. Time domain electromagnetic field computation with finite difference methods. International Journal of Numerical Modelling: Electronic Networks, Devices and Fields 9, 295-319 (1996).

79. Shimada, K. et al. Extremely flat transmission band of forward volume spin wave using gold and yttrium iron garnet. J. Phys. D: Appl. Phys. 50, 275001 (2017)

80. Nagai, K., Cao, Y., Tanaka, T. \& Matsuyama, K. Binary data coding with domain wall for spin wave based logic devices. J. Appl. Phys. 111, 07D130-133 (2012).

\section{Acknowledgements}

This work was partly supported by a PRESTO grant (No. JPMJPR1524) from the Japan Science and Technology Agency (JST) and by Grants-in-Aid for Scientific Research (KAKENHI) (Nos. 17K19029, 16H04329, and 26220902) from the Japan Society for the Promotion of Science (JSPS). T.G. acknowledges support from the Yazaki Memorial Foundation of Science and Technology, and MIC/SCOPE No. 192206001. C.A.R. acknowledges the support of NSF ECCS (Award No. 1607865). K.S. acknowledges the support from JSPS (KAKENHI, Nos. 25706004, 16H02098, and 16K13670). We also thank Mr. Akihiko Banno, Mr. Mei Goto, Prof. Yasushi Endo, and Prof. Takayuki Shibata for experimental support. We also acknowledge Shin-Etsu Chemical Co., Ltd. for their support.

\section{Author contributions}

T.G. planned the study and prepared and characterized the samples. T.Y. characterized the devices. K. Shimada, T.G. and B.I. calculated using a finite integration technique. T.G. and C.R. wrote the manuscript after discussions with A.G., K. Sekiguchi, Y.N., H.U. and M.I.

\section{Competing interests}

The authors declare no competing interests.

\section{Additional information}

Correspondence and requests for materials should be addressed to T.G.

Reprints and permissions information is available at www.nature.com/reprints. 
Publisher's note Springer Nature remains neutral with regard to jurisdictional claims in published maps and institutional affiliations.

(c) (i) Open Access This article is licensed under a Creative Commons Attribution 4.0 International License, which permits use, sharing, adaptation, distribution and reproduction in any medium or format, as long as you give appropriate credit to the original author(s) and the source, provide a link to the Creative Commons license, and indicate if changes were made. The images or other third party material in this article are included in the article's Creative Commons license, unless indicated otherwise in a credit line to the material. If material is not included in the article's Creative Commons license and your intended use is not permitted by statutory regulation or exceeds the permitted use, you will need to obtain permission directly from the copyright holder. To view a copy of this license, visit http://creativecommons.org/licenses/by/4.0/.

(c) The Author(s) 2019 